\title{
Reduction of Axial Kinetic Energy Induced Perturbations on Observed Cyclotron Frequency
}

\author{
Nathan K. Kaiser, Chad R. Weisbrod, Brian N. Webb, \\ and James E. Bruce \\ Department of Chemistry, Washington State University, Pullman, Washington, USA
}

\begin{abstract}
With Fourier transform ion cyclotron resonance (FTICR) mass spectrometry one determines the mass-to-charge ratio of an ion by measuring its cyclotron frequency. However, the need to confine ions to the trapping region of the ion cyclotron resonance (ICR) cell with electric fields induces deviations from the unperturbed cyclotron frequency. Additional perturbations to the observed cyclotron frequency are often attributed to changes in space charge conditions. This study presents a detailed investigation of the observed ion cyclotron frequency as a function of ion z-axis kinetic energy. In a perfect three-dimensional quadrupolar field, cyclotron frequency is independent of position within the trap. However, in most ICR cell designs, this ideality is approximated only near the trap center and deviations arise from this ideal quadrupolar field as the ion moves both radially and axially from the center of the trap. To allow differentiation between deviations in observed cyclotron frequency caused from changes in space charge conditions or differences in oscillation amplitude, ions with identical molecular weights but different axial kinetic energy, and thus amplitude of $z$-axis motion, were simultaneously trapped within the ICR cell. This allows one to attribute deviations in observed cyclotron frequency to differences in the average force from the radial electric field experienced by ions of different axial amplitude. Experimentally derived magnetron frequency is compared with the magnetron frequency calculated using SIMION 7.0 for ions of different axial amplitude. Electron promoted ion coherence, or EPIC, is used to reduce the differences in radial electric fields at different axial positions. Thus with the application of EPIC, the differences in observed cyclotron frequencies are minimized for ions of different axial oscillation amplitudes. (J Am Soc Mass Spectrom 2008, 19, 467-478) (C) 2008 American Society for Mass Spectrometry
\end{abstract}

$\mathrm{F}$ ourier transform ion cyclotron resonance (FTICR) mass spectrometry $[1,2]$ has become the ideal mass analyzer for the analysis of multiply charged ions produced by electrospray ionization with its ability to provide high-resolution and accurate mass measurements. It has become common practice to couple electrospray ionization to liquid chromatography for online analysis of complex mixtures with FTICRmass spectrometry (MS) [3-5]. To effectively sample the chromatographic peaks as they elute from the column, a short duty cycle is needed. However, with ion cyclotron resonance (ICR) mass spectrometers, the achievable mass resolving power, sensitivity, and mass measurement accuracy decrease with shorter acquisition time periods. Therefore, the majority of the time ions spent in the ICR cell is used for detection and not on some form of ion manipulation event such as ion accumulation, ion focusing, or ion selection. To meet

Address reprint requests to Professor James E. Bruce, Washington State University, Department of Chemistry, P.O. Box 644630, Pullman, WA 99164-4630. E-mail: james_bruce@wsu.edu the demand for high-throughput analysis while utilizing the high performance that FTICR mass spectrometers offer, ions are usually accumulated external to the magnetic field and then trapped in the ICR cell with some variation of gated trapping [3, 6-8]. Hybrid instruments allow for ion selection and fragmentation to take place external to the magnetic field. Minimizing individual spectrum acquisition time increases the number of MS and tandem mass spectrometry (MS/ MS) acquisitions that can be obtained during an liquid chromatography (LC)-MS analysis. Therefore, little time is spent on ion manipulation inside the ICR cell.

Ions are trapped in the ICR cell perpendicular $(x-y)$ to the cell axis by the magnetic field and along the ICR cell axis $(z)$ by a static potential applied to the trapping electrodes. The finite dimensions of the ICR cell result in two further motions in addition to cyclotron motion within the ICR cell: a trapping oscillation along the $z$-axis and magnetron motion. The ability to produce a three-dimensional (3D) axial quadrupolar potential is needed to obtain an ion cyclotron resonance frequency that is independent of the ion location within the ICR 
cell. This ideal electric field shape can be achieved only with a hyperbolic-shaped cell [9]. However, by applying the same dc voltage to each of the trapping electrodes of just about any trap geometry will approximate a quadrupolar electrostatic trapping potential near the center of the ICR cell [10]. This idealized electrostatic field is present only at the center of the ICR cell and, as the ions are moved axially and/or radially away from the ICR cell center, the electric field deviates from quadrupolar. Therefore, the observed cyclotron frequency becomes dependent on its position inside the ICR cell. The variation in cyclotron frequency as a function of $z$-axis position has been of concern to researchers in the ICR field for many years [11, 12]. Early trapped cell experiments by McIver and coworkers had hyperbolic-shaped electrodes to minimize these effects [13]. Many groups have shown that detailed knowledge of frequency shifts due to nonideal electric fields are required to maximize instrument performance [14-16]. Mitchell developed a theory of trapped ion motion in the non-quadrupolar electrostatic potential of a cubic ICR cell [17]. At a large radial or axial distance from the center of the ICR cell the rapid periodic cyclotron and axial motions of a single ion time-average spatial nonidealities in the electric and magnetic fields [18]. However, these same nonidealities also disrupt the ion motion of a coherent ion packet, increasing the rate of signal decay [19]. There are a number of trap configurations that have been proposed or tested to decrease these nonidealities [20-25].

With the 3D quadrupolar potential achieved at the center of the ICR cell it becomes desirable to reduce the ion oscillation amplitude so that ions are located primarily at the center of the ICR cell during excitation and detection. In addition, quadrupolar axialization improves virtually every aspect of FTICR performance [26]. Higher performance measurements are usually obtained with some form of ion cooling method as well as a reduction of trap plate potentials before ion detection $[27,28]$. There are a number of different methods that have been used to reduce ion axial motion in the ICR cell such as introduction of a collision gas into the ICR cell [29], side-kick [8], evaporative cooling [30, 31], and adiabatic cooling [27]. The method we describe here has great potential in combination with these methods to further minimize differences in magnetron frequency for ions of different $z$-axis oscillation amplitudes.

Gated trapping is attractive because it can decrease the data acquisition time period by accumulating ions external to the ICR cell. However, when gated trapping is performed ions can have different $z$-axis kinetic energies in the ICR cell. However, due to time-of-flight effects and the ion distribution as they exit the accumulation cell, all ions do not reach the center of the ICR cell at the same time [32]. The position of the ions at the moment the potential applied on the front plate is increased will result in different trapping oscillation amplitudes. Ions located at the center of the trap will be efficiently trapped. However, ions located at the edges of the trapping regions when the potential is increased will result in those ions having larger $z$-axis oscillation amplitudes. This produces a dispersion of ions along the $z$-axis of the ICR cell. This will result in a change in the observed cyclotron frequency based on ion axial position and/or rapid dephasing of the ion cloud [6, 19, 33, 34]. Therefore, scan-to-scan variation of ion kinetic energy will change the observed cyclotron frequency and also should cause nonlinear calibration errors. $z$-Axis motion is an important parameter to consider when performing high-performance measurements, since ions of different $z$-axis amplitude may experience different average magnetic field strength and radial electric field strength. It has been reported that during the detection period, the trapping motion effectively averages out differences in electric or magnetic field over $z$-axis amplitudes $[18,35]$. This is explained by motional averaging of the radial electric field. That is, the $z$-axis oscillation frequency is usually much higher than the magnetron frequency. Thus, motional averaging of the radial electric field during $z$-axis oscillation yields an average of the radial electric field experienced by an ion [36]. Vartanian and Laude [37] showed that for an open cylindrical cell modulation of the $z$-axis oscillation amplitude induced changes in the magnetron frequency.

Motion of ions in the ICR cell is complex and still not fully understood. Although fairly simple for a single ion, the complexity of ion motion increases with increasing number of ions within the ICR cell. Small perturbations in the observed cyclotron frequency from scan to scan are often attributed to changes in space charge conditions. This work focuses on changes in the observed cyclotron frequency as a function of $z$-axis oscillation amplitude. We show that ions with the same mass but different axial kinetic energies exhibit different observed cyclotron frequencies. We also present a further utility of the technique called electron promoted ion coherence (EPIC) [38-40], in which the application of an electron beam through the center of the ICR cell during detection alters the radial electric fields to produce longer transients. This technique is similar to the wire ion guide ICR cell designed by Russell and coworkers, which utilized a wire suspended along the axis of the ICR cell for modification of the trapping electric field [41, 42]. In our first EPIC paper we showed that with EPIC, we could achieve a threefold improvement in resolution and sensitivity by changing the electric fields. In the second EPIC paper we demonstrated that high mass measurement accuracy is possible with the technique, and characterized the effects the electron beam has on the observed cyclotron frequency. Here we present additional results that illustrate the underlying principle for the improvements made possible with this technique. In this research, the use of EPIC produces an observed cyclotron frequency that is independent of $z$-axis oscillation amplitude. 


\section{Experimental}

Ions were produced using electrospray ionization by applying approximately $2.5 \mathrm{kV}$ to the entrance to the mass spectrometer. The electrospray solution was 49 : 49:2 by volume of water:methanol:acetic acid. Ions were analyzed with a Bruker Daltonics 7T ApexQ FTICR mass spectrometer (Billerica, MA, USA). The instrument utilizes the Infinity cell [43] for image current detection. The mass spectral data were acquired with Xmass version 7.0.6 as the data acquisition software. Bradykinin, melittin, and insulin were purchased from Sigma (St. Louis, MO, USA). Solutions of peptides (10 $\mu \mathrm{M})$ were infused by direct injection with a syringe pump at a rate of $0.4 \mu \mathrm{L} / \mathrm{min}$. Ions were accumulated in a hexapole following isolation with the mass selective quadrupole. Ion intensity was varied by changing the ion accumulation time in the hexapole. The accumulation time varied between $0.1 \mathrm{~ms}$ and $2.0 \mathrm{~s}$. Ions were trapped in the ICR cell with the use of gated trapping. The ions were typically excited to cyclotron radius between 30 and $40 \%$ of the ICR cell radius. This radius corresponds to the inflection point in the radial electric fields derived from the SIMION modeling for EPIC experiments. The data were analyzed with ICR-2LS [44]. To determine frequencies, all transients were Welch apodized followed by one zero-fill before Fourier transformation to the frequency domain. Ion abundances used for space charge frequency corrections were calculated by taking the initial amplitude from the extracted transient of the monoisotopic peak in the frequency domain $[45,46]$. This process was done instead of obtaining ion abundances directly from the peak intensity because the peak intensity will change for ions with different signal decay rates.

The pulse sequence to simultaneously trap ions with different axial kinetic energies in the ICR cell called a "double trap" experiment is shown in Figure 1a. Bradykinin $(\mathrm{M}+2 \mathrm{H})^{2+}$ was accumulated in the hexapole region and then sent to the ICR cell followed by injection of a cooling gas (argon) with a pulse length of $1.5 \mathrm{~ms}$. After an 8-s delay, a second ion packet of bradykinin $(\mathrm{M}+2 \mathrm{H})^{2+}$ is accumulated in the hexapole and sent to the ICR cell, followed immediately by excitation and detection. The pulse sequence was set up to individually control the ion accumulation time period for each ion injection event. The trapping potentials were set at $1.5 \mathrm{~V}$ for all "double trap" experiments.

The magnetron frequency was calculated with SIMION 7.0 software (SIMION 7.0 3D, version 7.0, D. A. Dahl, Idaho National Engineering Laboratory, Idaho Falls, ID). The magnetic field strength was set at 7 Tesla for the magnetron frequency simulations. The ICR cell used for the simulations was a closed cylindrical cell (dimensions: $64 \mathrm{~mm}$ long; diameter: $60 \mathrm{~mm}$ ). The radial electric fields were also calculated with SIMION 7.0 at 1 -mm intervals along the $z$-axis, at different cell radii. The segmented trap plates of the Infinity cell were treated as if they were a single electrode. This will cause only minor deviations in the calculations very near the

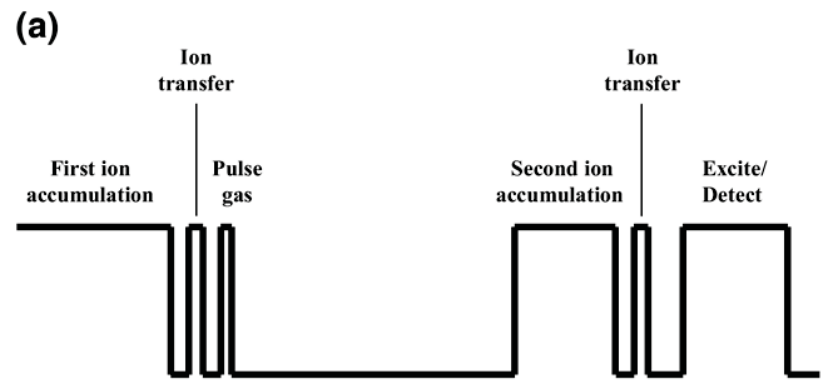

(b)

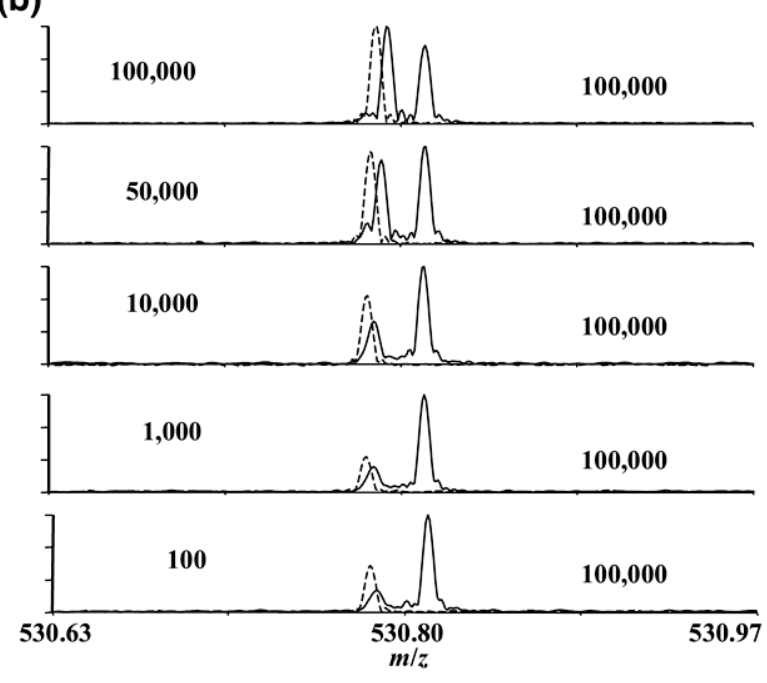

(c)

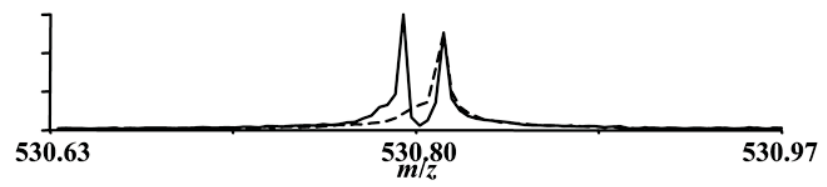

Figure 1. "Double trap" experiment. (a) Pulse sequence for the "double trap" experiment that includes two separate ion injection events. (b) The monoisotopic peak of bradykinin $(\mathrm{M}+2 \mathrm{H})^{2+}$ with different ion accumulation time periods given in microseconds. Solid line represents the "double trap" experiment. The first ion accumulation time period (located left of the peak) was varied, whereas the second ion accumulation time period (located to the right of the peak) remained constant. The dotted line represents a single ion injection that corresponds to the first ion injection event (cooled ion packet) of the "double trap" experiment. The accumulation for the single ion injection experiment was also varied. (c) The solid line is the "double trap" experiment and the dotted line is a single ion injection that corresponds to the second ion injection event of the double trap experiment.

surface of the electrodes, and would not alter the calculated fields presented here.

To initiate larger $z$-axis amplitude, ions were first cooled axially with a pulse gas valve event; $1.5 \mathrm{~V}$ was applied to both trap plates. After an 8-s delay the back trap plate was dropped to ground for a defined period of time (51 to $2000 \mu \mathrm{s}$ ), then raised back to $1.5 \mathrm{~V}$ for 400 $\mu \mathrm{s}$, before being dropped back to ground. The front trap plate remained at $1.5 \mathrm{~V}$. The number of times that the back trap plate was lowered and raised was varied between 1 and 10 . 
EPIC was carried out with a hollow cathode originally installed to conduct electron capture dissociation experiments. The cathode was heated with 1.5 to 1.6 amps of current. To send electrons through the center of the ICR cell during detection the potential to the cathode was pulsed negative $(0.0$ to $-1.0 \mathrm{~V})$. At all other times the potential applied on the cathode was held at a positive $10 \mathrm{~V}$. The cathode is pulsed negative after the excitation event and remained negative throughout the detection event.

\section{Results and Discussion}

\section{Simultaneous Detection of Ions with Different Kinetic Energies}

Although it has been shown that motional averaging of the radial electric field results in a single observed cyclotron frequency for a range of different axial kinetic energies, it is possible to observe separate peaks within the same spectrum for ions with the same mass value but distinctly different axial kinetic energies. Figure $1 b$ shows the results from the double trap experiment in which the first ion packet is injected into the ICR cell followed by a pulse gas event. This was done to cool the axial motion and allow the ions to be focused axially to the center of the ICR cell. After an 8-s delay to allow the cooling gas to be pumped away, a second ion packet is injected into the ICR cell. This allows for two packets of ions with the exact same mass value that differ only in kinetic energy to be trapped in the ICR cell. The cooled ions will have small z-axis oscillation amplitudes, whereas the ions from the second injection will have much larger $z$-axis oscillation amplitude. The resulting mass spectrum yields two peaks for the same mass value. In Figure 1b, the accumulation time for the first ion injection event was varied, whereas the accumulation time for the second ion injection event remained constant. As expected, increasing the accumulation time for the first ion injection event results in an increase in peak intensity for the corresponding peak in the spectra, whereas the peak intensity resultant from the second ion injection event remained constant. This demonstrates independent control over the number of ions injected into the ICR cell for the separate ion injection events. The results from the double trap experiment were compared with a single ion injection event that mimicked either the first or second ion injection event of the double trap experiment. The result from only a single ion injection for the first injection event is represented by the dotted line in Figure 1b. The result for the single ion injection for the second ejection event is shown in Figure 1c. The pulse sequence remained the same between the single ion injection event and the double trap experiment, with the exception of altering a voltage parameter in the source region to gate the ions. For the same ion accumulation times, the peak intensities are similar between the single ion injection and the corresponding peak in the double trap experiment. The peak for the single injection is shifted to a slightly lower $\mathrm{m} / \mathrm{z}$ value, indicating higher frequency. This could result from a lower number of total ions trapped in the ICR cell (one ion injection versus two ion injections) that results in different space charge conditions. Alternatively, the z-axis oscillation amplitude of the cooled ion packet may have increased slightly during the injection of the second ion packet. The front trap plate was pulsed to ground for the ion flight time $(1.5 \mathrm{~ms})$ before being raised to $1.5 \mathrm{~V}$ for ion detection.

The double trap experiment was designed to minimize differences in space charge conditions that might occur if the experiments were performed separately. If the frequencies had been measured in separate experiments it would be difficult to differentiate frequency shift contributions to the observed cyclotron frequency resultant from space charge from those resultant from axial oscillation amplitude. The double trap experiments allow us to ascribe the differences in observed frequencies to axial oscillation amplitude differences. In reducing the axial kinetic energy of the ions in the ICR cell with a collision gas, the space charge conditions are also changing. A cooled ion packet likely experiences higher space charge conditions since the spread in axial positions of the ions is decreased. One might therefore expect cooled ions to exhibit lower cyclotron frequencies due to increased space charge conditions [47, 48]. However, as shown in Figure $1 \mathrm{~b}$ the cooled ion packet is observed at a lower $m / z$ value, indicating that it has a higher observed cyclotron frequency. The difference in frequency results from the difference in average radial electric field experienced by the ions. Figure 2 shows the calculated magnitude of the outward directed radial electric field along the $z$-axis at $45 \%$ of the ICR cell radius with $1 \mathrm{~V}$ applied to the trap electrodes. The magnitude of the radial electric field changes with ion position along the $z$-axis. Increasing the trap plate voltage will increase the difference in magnitude of the radial electric field across the $z$-axis of the ICR cell. Therefore, as an ion oscillates between the trap plates it will experience differences in the magnitude of the radial electric field. The resultant magnetron frequency is correlated to the average radial electric field experienced by the ions. Therefore, changes in the average radial electric field produced from differences in axial motion will be reflected in the magnetron frequency.

The magnetron frequency is also shown in Figure 2 for ions with different axial oscillation amplitudes. Ions with different axial kinetic energy in the ICR cell will have different axial oscillation amplitudes, and will thus experience a different average radial electric force. Dunbar and coworkers [49] were able to map out electric field imperfections in a cubic ICR cell by monitoring the magnetron frequency of the ions. A cooled ion packet will have small axial oscillation amplitude, will be located at the center of the ICR cell, and will experience little change in the radial electric field along the $z$-axis. In Figure 2, the magnetron frequency is smallest at the center of the ICR cell. As the kinetic 


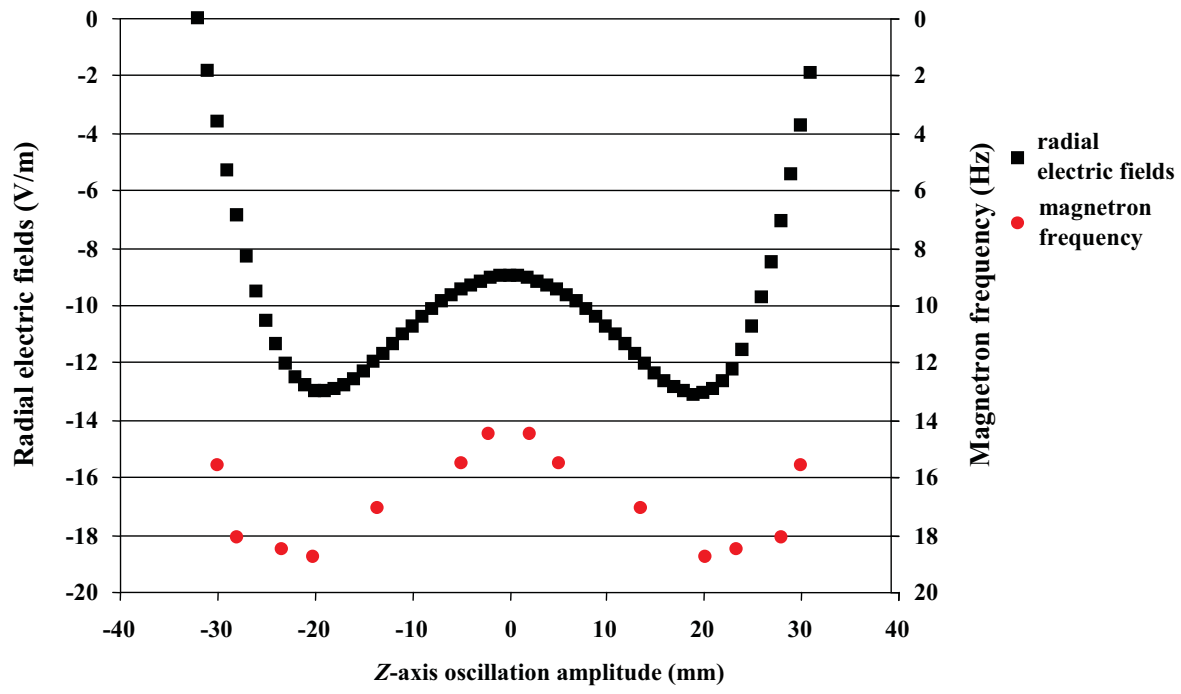

Figure 2. SIMION calculated radial electric fields and magnetron frequency at $45 \%$ of the ICR cell radius with $1 \mathrm{~V}$ applied to the trap electrodes. The center of the ICR cell is set at $0 \mathrm{~mm}$.

energy or amplitude of z-axis motion increases, the magnetron frequency increases. An ion with small amplitude of $z$-axis motion will have a greater observed cyclotron frequency because it has a smaller magnetron frequency. This relationship is shown in Eq 1 where $\omega_{+}$ is the observed cyclotron frequency, $\omega_{c}$ is the unperturbed cyclotron frequency, and $\omega_{-}$is the magnetron frequency [1]:

$\omega_{+}=\omega_{c}-\omega_{-}$
Effect of Ion Cooling on Observed Cyclotron Frequency

Ion kinetic energies were varied to observe the effect of different axial oscillation amplitude on observed cyclotron frequency. Ions are cooled either through a pulsed gas event or by the addition of a significant delay between ion injection into the ICR cell and excitation. Results for the experiments are shown in Figure 3. The highest observed cyclotron frequency occurs after the

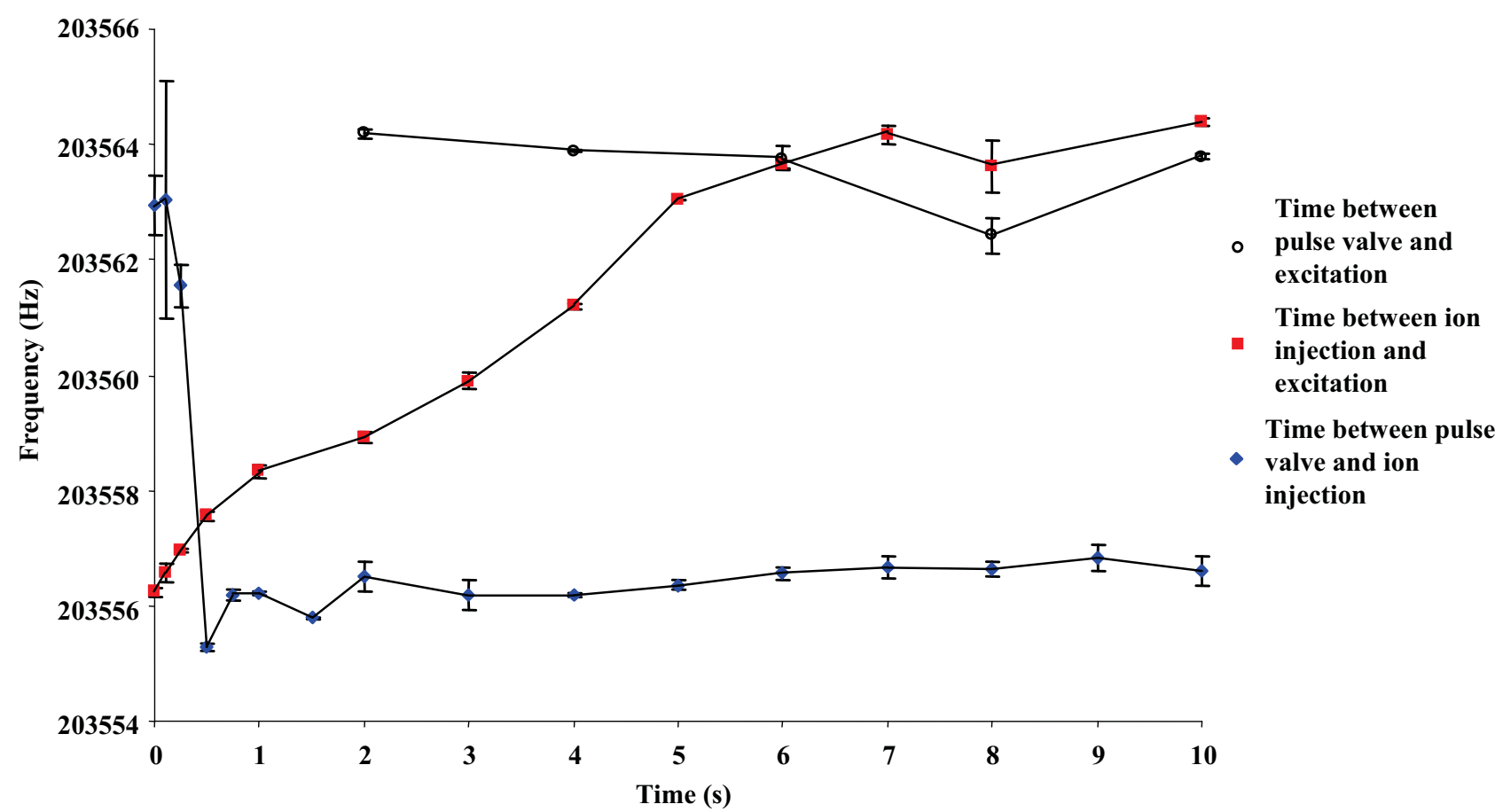

Figure 3. Observed cyclotron frequency of the monoisotopic peak from bradykinin $(\mathrm{M}+2 \mathrm{H})^{2+}$ with different time delays between events. The error bars represent the maximum and minimum values obtained for each dataset. 
ions have been cooled to the middle of the ICR cell. Three sets of data are illustrated in this figure. The duration between the pulse valve event following ion injection and cyclotron excitation $(\bigcirc)$ was varied. Increasing the duration between the pulse event showed no noticeable trend in measured cyclotron frequencies. This indicates that ions are cooled axially to the middle of the ICR cell. No time points were taken with a delay of $\leq 2 \mathrm{~s}$ due to the deleterious effects the high pressure has on ICR signal detection. Thus, the delay period between ion cooling and ion excitation had no observable effect on the axial position of the ions. The next set of experiments examined the frequency difference for ions that were subjected to variable delay times between ion injection into the ICR cell and cyclotron excitation with no pulsed valve ( $\mathbf{\square})$. With a delay of about $10 \mu \mathrm{s}$ between ion injection and excitation the ions have the lowest observed frequency, which indicates that these ions have the largest axial motion immediately after ion injection. As the delay period increases the observed frequency increases, which results from ions being cooled to the middle of the ICR cell through either ion-neutral or ion-ion interactions. The signal amplitude remained constant for each delay period, indicating that ions are not lost from the ICR cell during the delay period. Therefore, the change in frequency can be attributed to a change in axial position, rather than alterations in space charge conditions. After approximately a 7-s delay the observed frequency leveled off at the same frequency one would expect if the ions were cooled to the center of the ICR cell with the addition of a cooling gas. This indicates two things: first, after some set time period the axial oscillation amplitude was not being reduced further and, second, the ion axial amplitude with the delay between ion injection and excitation approaches the ion axial amplitude following the pulse gas event. This experiment was also carried out with melittin and insulin to test for mass dependence of this axial relaxation. All three tested species exhibited the same effect of increased frequency with delay time before leveling off at observed frequencies that agreed with those recorded with the corresponding pulse gas experiments. Figure 3 also shows results of the experiments in which a pulse gas event was followed by a variable delay time before ion injection into the ICR cell $(\diamond)$. When the pulse gas event is immediately followed by ion injection, there are enough neutral molecules in the ICR cell to cool the ions the same as if the ions were trapped and cooled with a pulse gas event. This is evident from the data in Figure 3 because the measured frequencies with this delay period of $\leq 0.5 \mathrm{~s}$ agreed closely with those measured for cooled ions $(\bigcirc)$. It should be noted that, if the delay period between the pulse gas event and ion injection was $<2 \mathrm{~s}$ an additional delay was added so that the total time between the pulse gas and cyclotron excitation was $>2 \mathrm{~s}$ to allow time for the residual neutral molecules to be pumped away. As the delay period between the pulse gas and ion injection increases, the observed frequency decreases. This results from the neutral gas molecules being pumped away and, therefore, when ions reach the ICR cell there are insufficient ion-neutral collisions to promote complete cooling of axial ion motion. Furthermore, a delay of $\geq 1 \mathrm{~s}$ results in observed frequencies that are in close agreement with those acquired with no pulsed valve (回). Importantly, these data illustrate that the degree to which the ions are cooled changes their axial oscillation amplitude and thus their observed frequencies.

\section{Determination of Magnetron Frequency}

Magnetron frequency was determined experimentally for both the pulsed gas and the non-pulsed gas experiments. This was achieved by varying the trap plate potentials from 3.0 to $0.5 \mathrm{~V}$. The application of trap plate potentials induces the radial electric field that drives magnetron motion. Increasing the applied trap plate potentials increases the force from the outward directed radial fields and results in higher magnetron frequencies. The results from investigation of the effects of trap potentials on observed frequencies with and without pulsed gas both produced linearly increasing frequencies with decreasing trap plate potentials. The observed frequencies for the cooled ions were higher at every applied trap plate potential. The difference between the two datasets becomes greater with increased trap plate potential. The observed cyclotron frequencies for the non-pulsed gas experiments decreases (as trap potentials are increased) at a greater rate. This indicates that the radial electric fields are changing more at greater $z$-axis position with increased trap plate potentials. Extrapolation of the measured cyclotron frequency to 0 $\mathrm{V}$ on the trapping plates produces the unperturbed cyclotron frequency. With $0 \mathrm{~V}$ applied to the trapping plates, the ions will not experience any radial fields and there should be an absence of magnetron motion. The difference between the $y$-intercept and the observed cyclotron frequency is the result of the magnetron frequency in the absence of space charge conditions. Differences in ion intensity at the different trap plate potentials were corrected with the method developed by Easterling and colleagues [50]. Separate ion intensity calibration curves were constructed for the pulsed gas and non-pulsed gas experiments. Each frequency was corrected by adding the correct shift (based on ion intensity) to obtain a frequency with minimal space charge conditions. The two experiments have different calculated magnetron frequencies that result from differences in the $z$-axis oscillation amplitudes. As the trap plate potentials increase, the difference in magnetron frequency increases. The calculated magnetron frequency for the experiments performed without cooling gas increases at a rate of $18.1 \mathrm{~Hz} / \mathrm{V}$ at an intercept of $-0.51 \mathrm{~Hz}$, and for the pulsed gas experiments the magnetron frequency increases at a rate of $15.0 \mathrm{~Hz} / \mathrm{V}$ with an intercept of $0.08 \mathrm{~Hz}$. This result is compared with the magnetron frequency calculated 
with SIMION 7.0. The magnetron frequency is calculated with $z$-axis amplitude of $2 \mathrm{~mm}$ and a radius of $10 \mathrm{~mm}$ to compare with the pulsed gas event, and also with z-axis amplitude of $38 \mathrm{~mm}$ and a radius of $10 \mathrm{~mm}$ to compare to the non-pulse gas experiment. The radius of $10 \mathrm{~mm}$ is chosen to match the calculated excited cyclotron radius; ions will experience the radial electric force at the position in which they are located in the ICR cell. The experimental and calculated magnetron frequencies match closely, indicating that ions of different $z$-axis oscillation amplitudes have different magnetron frequencies.

At $1 \mathrm{~V}$ trap potential, the frequency difference between the two experiments is about $2.5 \mathrm{~Hz}$. A 1- to $2-\mathrm{Hz}$ shift in frequency will result in a 9-18 ppm error for an $\mathrm{m} / \mathrm{z}$ value of 1000 for an FTICR instrument equipped with a 7 Tesla magnet. Ions that do not have the same ion kinetic energy from scan to scan will have a slight variation in the observed cyclotron frequency. Thus, degradation in the observed mass measurement accuracy is expected under these conditions. Also, one might expect ions of the same cyclotron frequency but different magnetron frequency to dephase more rapidly. A 1 - to $2-\mathrm{Hz}$ difference indicates that ions with extreme differences in $z$-axis motion $(2-38 \mathrm{~mm})$ will be $180^{\circ}$ out of phase within $0.5-0.25 \mathrm{~s}$. This is in general agreement with experimental observations. The magnetron frequency decreases proportionally with increased magnetic field strength; thus the difference in magnetron frequency resulting from different oscillation amplitudes also decreases. Doubling the magnetic field strength should double the time it takes for ions to become $180^{\circ}$ out of phase. Therefore, working with higher field magnets will help, but ultimately not circumvent these problems. Improved performance must involve further ICR cell technology development.

\section{Increased z-Axis Amplitude through Excitation of Trapping Motion}

The $z$-axis motion of a cooled ion packet was excited to further probe the effect of $z$-axis distribution on observed ion cyclotron frequency. The z-axis motion was excited by first cooling the ion packet to the middle of the ICR cell with cooling gas followed by dropping the back trap plate to ground successive times for a total of 10 cycles. The time period that the back trap plate was held at ground was varied. This was done to excite the $z$-axis motion and move the ions from the middle of the trap. Figure 4 illustrates the results observed by varying the duration of the "pulse length" to ground on the back trap plate. When the back trap plate potential was dropped for a time period that approximated one period of trapping motion, the ions changed their axial distribution. The calculated trapping oscillation period for bradykinin $(\mathrm{M}+2 \mathrm{H})^{2+}$ with $1.5 \mathrm{~V}$ trapping potential was $0.323 \mathrm{~ms}$. If the "pulse length" was too long or too short the observed cyclotron frequency shifted back to the original frequency. The frequency of the trap plate oscillation was estimated to be one half the trapping frequency when the pulse length matched the trapping oscillation period. Therefore, at this pulse length it is possible to directly excite the axial motion and thus to increase the amplitude of ion axial oscillation. The total ion intensity shown in Figure 4 remained relatively constant, indicating that ions are not being ejected from the ICR cell when the trap plate was dropped to ground. However, if the time period that the back trap plate was dropped was too long ( $\gtrsim 2 \mathrm{~ms}$ ) the ion intensity decreased. This result agrees with our previous results that showed that ions with larger axial kinetic energies have lower observed cyclotron frequencies. The frequency shift observed in the experiment when the ions are given larger $z$-axis amplitude is about $4.5 \mathrm{~Hz}$ lower frequency. The magnetron frequency difference calculated with SIMION 7.0 for an ion with $z$-axis amplitudes of $2-38 \mathrm{~mm}$ at a $10-\mathrm{mm}$ radius with $1.5 \mathrm{~V}$ applied trapping potential was about $4.1 \mathrm{~Hz}$. The experimental results compare well with the calculated value. The decrease in the observed cyclotron frequency by increasing the $z$-axis amplitude results from a change in the magnetron frequency.

\section{EPIC Results}

EPIC was carried out to determine the effect it has on the observed cyclotron frequency as a function of z-axis oscillation amplitude. For the SIMION model, a solid electrode was used to approximate the electron beam. Although it is not an exact solution, the model does provide a first-order approximation of the electric fields inside the ICR cell with EPIC and agrees well with our previous results [40]. Possibly the large numbers of electrons in the beam behave more like a negatively charged plasma and occupy the space between the cathode and the source trap electrode of the ICR cell.

The application of EPIC flattens the radial electric field across the $z$-axis of the ICR cell at some non-zero cell radius. The radius at which EPIC results in minimal change in radial field across the $z$-axis is a function of the applied trap plate potential and the number of electrons traversing the ICR cell. The ability to produce invariable radial electric fields along the $z$-axis with EPIC is shown in Figure 5 [40]. The trapping potential was set to $1 \mathrm{~V}$ on both trapping electrodes. The electrostatic potential was calculated at $1-\mathrm{mm}$ intervals at $42 \%$ of the cell radius with EPIC (Figure 5a) and under normal operation (Figure 5b). Radial electric fields are also calculated and overlaid on top of the electrostatic trapping potentials. The ion oscillation length is also shown for two different ion kinetic energies. In the non-EPIC case (Figure 5b), there is a larger difference in the radial electric force experienced by ions with different kinetic energies. With EPIC the ions of different kinetic energies will experience similar radial electric 

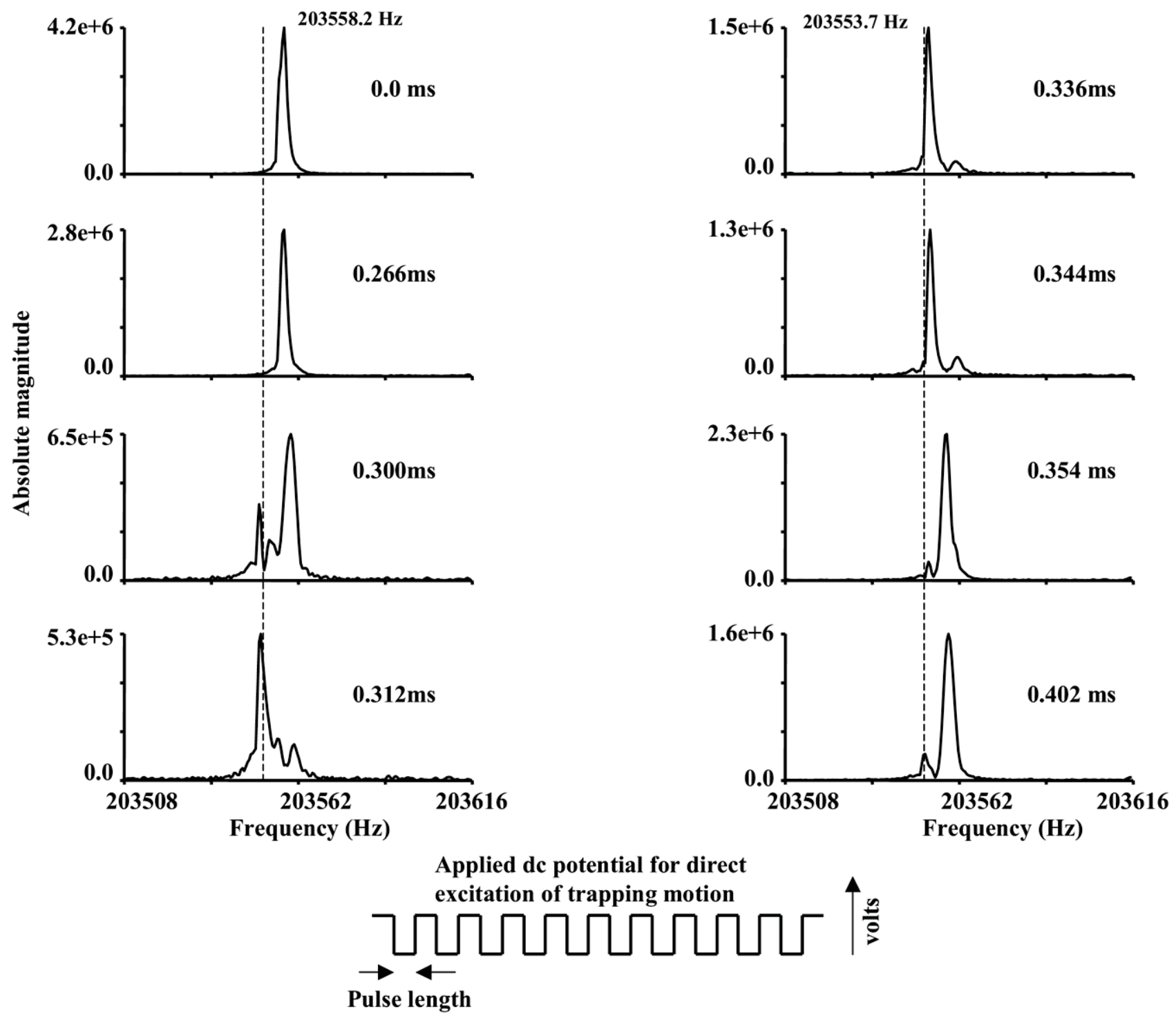

\begin{abstract}
Figure 4. Observed cyclotron frequency of bradykinin $(\mathrm{M}+2 \mathrm{H})^{2+}$ after $z$-axis excitation. Ions are first cooled with a pulse gas event, and then displaced axially by dropping the back trap plate 10 times. The time given represents the "pulse length" or time period the back trap plate was dropped to ground. The dashed line represents the same frequency in both columns and is added to provide a reference point for the frequency shift.
\end{abstract}

fields. When the ion cyclotron radius is excited to this particular cell radius, the ions will experience the same radial force regardless of their axial distribution. Therefore, magnetron frequency and thus the observed cyclotron frequency will become independent of $z$-axis amplitude.

EPIC was carried out with a variety of applied cathode bias potentials that change the numbers of electrons that transverse the ICR cell for both the pulse gas and non-pulsed gas experiments individually. The changes in detected cyclotron frequency observed with changing EPIC conditions for pulsed gas and nonpulsed gas experiments are shown in Figure 6a. With 0 $\mathrm{V}$ applied to the heated cathode, the difference in observed frequency between the two experiments is about $6 \mathrm{~Hz}$. This difference in frequency was consistent throughout all of our experiments, with the pulse gas having a higher frequency than that of the non-pulse gas experiment. This also indicates that there were not enough electrons being sent through the center of the ICR cell to significantly alter the radial electric fields. As the cathode potential is biased more negatively, the observed cyclotron frequency increases for both experiments. The rates at which the frequencies increase for the two sets of experiments are different. This indicates that the radial electric field is changing greater at a further distance from the center of the ICR cell. At about $-0.38 \mathrm{~V}$ bias to the cathode, there is no difference in the observed cyclotron frequencies between the two different experiments. This signifies that with the application of EPIC, the radial electric fields are constant across the ICR cell at the excited cyclotron radius of the ions. If 

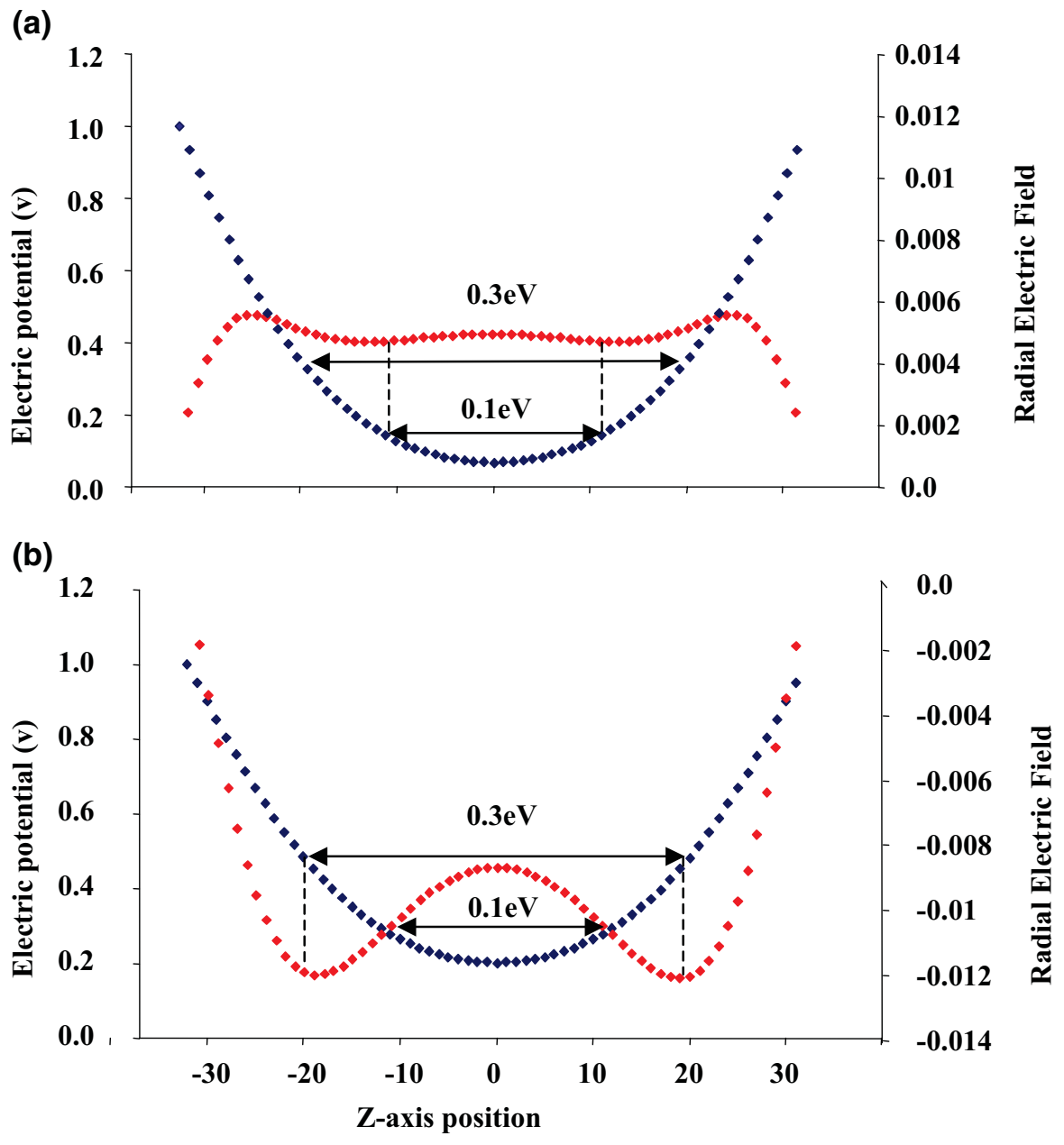

Figure 5. SIMION calculations of the radial electric field and electric potential at $42 \%$ of the ICR cell radius. (a) EPIC with $-0.2 \mathrm{~V}$ applied to the central electrode and $1 \mathrm{~V}$ applied to the trap electrodes. (b) Closed cylindrical cell with $1 \mathrm{~V}$ applied to the trap electrodes.

the cathode potential is further increased (negatively), the two frequencies diverge. The observed frequency with the non-pulse gas becomes greater than that of the pulse gas experiment. The increase in frequency results from the change in magnitude and direction of the radial electric field experienced by the ions. This indicates that with careful adjustment of the number of electrons sent through the ICR cell by tuning applied cathode voltage or heating current, the observed cyclotron frequency is independent of the $z$-axis oscillation amplitude of the ions.

To further test the effect EPIC has on the observed cyclotron frequency with z-axis oscillation amplitude the "double trap" experiment was conducted with EPIC. The results are shown in Figure $6 \mathrm{~b}-\mathrm{e}$. In the non-EPIC conditions there are two separate peaks that are visible in the spectrum for the "double trap" experiment, as would be expected based on the results from Figures $1 \mathrm{~b}$ and $6 \mathrm{a}$. Results from the cooled and uncooled single ion injection experiments are overlaid for reference of peak position. With $-0.4 \mathrm{~V}$ applied to the cathode there is only one peak that is visible for the "double trap" experiment. This corresponds to the data from Figure 6a, where the ions from the separate ion injection events exhibited the same observed frequencies. The frequency from the single ion injection experiments with EPIC match with the double ion injection experiments with EPIC. The ion intensity for the EPIC result in the "double trap" experiment is higher than that of the single ion injection, since the "double trap" experiment had more ions resulting from two different ion injection events. This shows that with EPIC there is no change in observed cyclotron frequency between cooled and uncooled ions. This is especially important for gated trapping when trapped ions may have differences in trapping oscillation amplitudes.

In our previous publication, we showed that the application of EPIC can improve the resolution and sensitivity by about threefold. Even after the ions were cooled with a collision gas and the trap plates set at low voltage potentials, we were still able to see these same improvements. In the double trap experiments, ions were forced to have different kinetic energies to allow us to study the change in frequency resulting from 
(a)

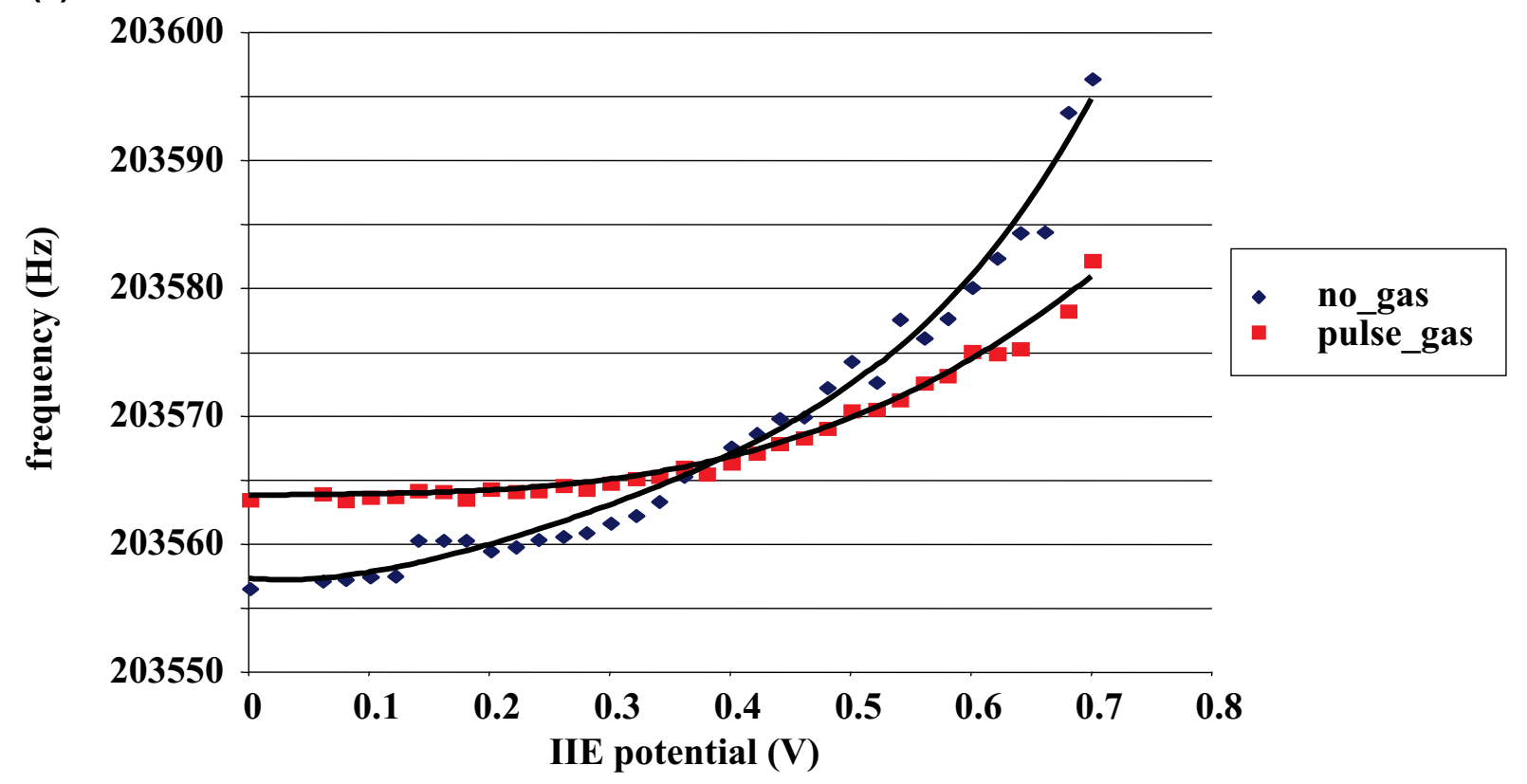

(b)

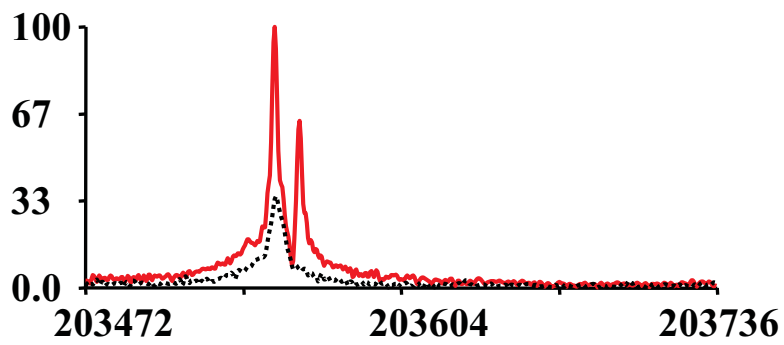

(d)

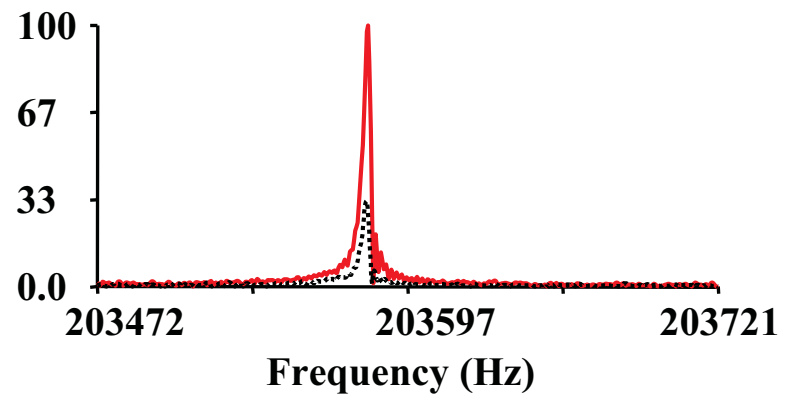

(c)

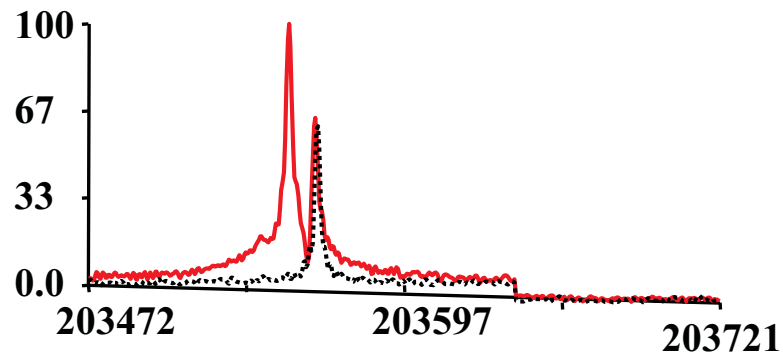

(e)

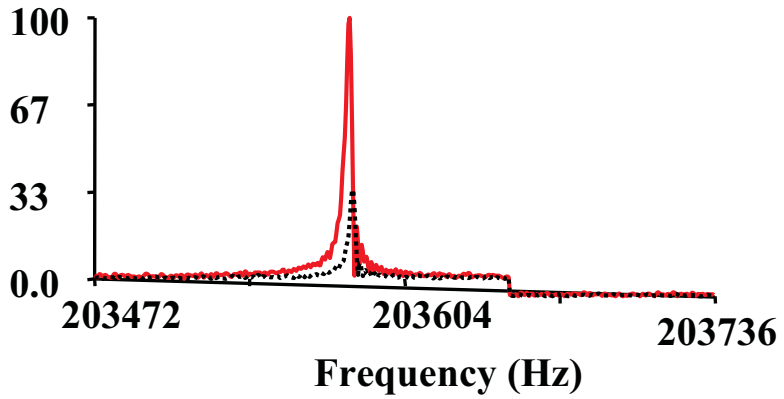

Figure 6. EPIC experiments. (a) Comparison of the observed cyclotron frequency for the monoisotopic peak of bradykinin $(\mathrm{M}+2 \mathrm{H})^{2+}$ for the pulsed gas and non-pulsed gas experiment with EPIC at different bias potentials applied to the heated cathode. (b) Non-EPIC conditions; the dotted line represents the second ion injection event only. (c) Non-EPIC conditions; the dotted line represents the first ion injection only. (d) Cathode potential set at $-0.4 \mathrm{~V}$; the dotted line represents the second ion injection event only. (e) Cathode potential set at $-0.4 \mathrm{~V}$; the dotted line represents the first ion injection event only. The "double trap" experiment is indicated by the solid line; the single ion injection experiment is indicated by the dotted line.

$z$-axis oscillation amplitude. Typically, an instrument is tuned to minimize large differences in axial kinetic energy. If the instrument is not properly tuned, this energy distribution may lead to peak splitting and rapid dephasing of the ion packet. However, in all cases, a distribution of $z$-axis kinetic energies is, to some extent, unavoidable. This is important because these ions will have slightly different reduced cyclotron frequencies that will not result in observation of multiple peaks in the mass spectrum, but rather, a decrease in ion intensity due to increased dephasing of ion cyclotron motion. EPIC reduces the radial electric field 
variations encountered by ions with a distribution of $z$-axis kinetic energies and can thus improve sensitivity, resolution, and mass accuracy by decreasing this dephasing mechanism as we have observed.

\section{Conclusions}

The observed cyclotron frequency for ions that have been cooled to the center of the ICR cell was higher than that of ions with larger $z$-axis oscillation amplitudes. Space charge effects did not appear to have a significant impact on the measured ion cyclotron frequency. However, the dominant divergence in observed cyclotron frequency between ion packets of distinctly different kinetic energy can be attributed to the difference in axial oscillation amplitude of the ions along the center of the ICR cell. Bradykinin $(\mathrm{M}+2 \mathrm{H})^{2+}$ ions of different axial kinetic energies were detected in the same spectrum to minimize differences that are associated with changing space charge conditions. At the excited ion cyclotron radii, the ion will experience differences in the radial electric field as it transverses the ICR cell. These differences become larger as differences in the ion kinetic energy increase. Since the force from the radial electric field produces magnetron motion, the magnetron frequency will change at different axial positions. Ions of different axial amplitude exhibit different magnetron frequencies that were determined experimentally and correlated well with the theoretical magnetron frequencies calculated in SIMION 7.0. Changes in magnetron frequency produce differences in observed cyclotron frequency. With the application of EPIC, changes in the radial electric field across the $z$-axis of the ICR cell at a selected cyclotron radius can be minimized. Therefore, as the ions of different kinetic energy oscillate along the $z$-axis of the ICR cell, they will experience little change in the radial electric field. Thus, the observed cyclotron frequency becomes independent of $z$-axis position and dephasing mechanisms resulting from $z$-axis-dependent changes in magnetron frequency can be minimized.

\section{Acknowledgments}

This material is based on work supported by the National Science Foundation under Grant 0352451; Murdock Charitable Trust; Office of Science (BER), U.S. Department of Energy Grant DEFG02-04ER63924; and a National Institutes of Health Biotechnology Training Grant.

\section{References}

1. Marshall, A. G.; Hendrickson, C. L.; Jackson, G. S. Fourier Transform Ion Cyclotron Resonance Mass Spectrometry: A Primer. Mass Spectrom. Rev. 1998, 17, 1-35.

2. Comisarow, M. B.; Marshall, A. G. Fourier Transform Ion Cyclotron Resonance Spectroscopy. Chem. Phys. Lett. 1974, 25, 282-283.

3. Senko, M. W.; Hendrickson, C. L.; Emmett, M. R.; Shi, S. D. H.; Marshall, A. G. External Accumulation of Ions for Enhanced Electrospray Ionization Fourier Transform Ion Cyclotron Resonance Mass Spectrometry. J. Am. Soc. Mass Spectrom. 1997, 8, 970-976.

4. Sharma, S.; Simpson, D. C.; Tolic, N.; Jaitly, N.; Mayampurath, A. M.; Smith, R. D.; Pasa-Tolic, L. Proteomic Profiling of Intact Proteins Using WAX-RPLC 2-D Separations and FTICR Mass Spectrometry. J. Proteome Res. 2007, 6, 602-610.
5. Belov, M. E.; Anderson, G. A.; Wingerd, M. A.; Udseth, H. R.; Tang, K.; Prior, D. C.; Swanson, K. R.; Buschbach, M. A.; Strittmatter, E. F.; Moore, R. J.; Smith, R. D. An Automated High Performance Capillary Liquid Chromatography-Fourier Transform Ion Cyclotron Resonance Mass Spectrometer for High-Throughput Proteomics. J. Am. Soc. Mass Spectrom. 2004, 15, 212-232.

6. Gorshkov, M. V.; Masselon, C. D.; Anderson, G. A.; Udseth, H. R.; Harkewicz, R.; Smith, R. D. A Dynamic Ion Cooling Technique for FTICR Mass Spectrometry. J. Am. Soc. Mass Spectrom. 2001, 12, 1169 1173.

7. Alford, J. M.; Williams, P. E.; Trevor, D. J.; Smalley, R. E. Metal Cluster Ion Cyclotron Resonance. Combining Supersonic Metal Cluster Beam Technology with FT-ICR. Int. J. Mass Spectrom. Ion Process. 1986, 72, 33-51.

8. Caravatti, P. Method and Apparatus for The Accumulation of Ions in a Trap of an Ion Cyclotron Resonance Spectrometer, by Transferring the Kinetic Energy of the Motion Parallel to the Magnetic Field into Directions Perpendicular to the Magnetic Field. U.S. Patent 4924 089, 1990.

9. Marto, J. A.; Marshall, A. G.; Schweikhard, L. A Two-Electrode Ion Trap for Fourier Transform Ion Cyclotron Resonance Mass Spectrometry. Int. J. Mass Spectrom. Ion Process. 1994, 137, 9-30.

10. Guan, S.; Marshall, A. G. Ion Traps for Fourier Transform Ion Cyclotron Resonance Mass Spectrometry: Principles and Design of Geometric and Electric Configurations. Int. J. Mass Spectrom. Ion Process. 1995, 146/147, 261-296.

11. McIver, R. T., Jr.; Baranyi, A. D. High Resolution Ion Cyclotron Resonance Spectroscopy. Int. J. Mass Spectrom. Ion Phys. 1974, 14, 449-458.

12. Marshall, A. G. Theory for Ion Cyclotron Resonance Absorption Line Shapes. J. Chem. Phys. 1971, 55, 1343-1354.

13. McIver, R. T., Jr.; Ledford, E. B., Jr.; Miller, J. S. Proposed Method for Mass Spectrometric Analysis for Ultra-Low Vapor Pressure Compounds. Anal. Chem. 1975, 47, 692-697.

14. Brown, L. S.; Gabrielse, G. Geonium Theory: Physics of a Single Electron or Ion in a Penning Trap. Rev. Modern Phys. 1986, 58, 233-311.

15. Holliman, C. L.; Rempel, D. L.; Gross, M. L. A Mechanism for Poor High Mass Performance in Fourier Transform Mass Spectrometry. J. Am. Soc. Mass Spectrom. 1992, 3, 460-463.

16. Hartmann, H.; Chung, K. M.; Baykut, G.; Wanczek, K. P. Dependence of Ion Cyclotron Frequency on Electric Field Inhomogeneity. J. Chem. Phys. 1983, 78, 424-431.

17. Mitchell, D. W. Theory of Trapped Ion Motion in the Non-Quadrupolar Electrostatic Potential of a Cubic Ion Cyclotron Resonance Cell. Int. J. Mass Spectrom. Ion Process. 1995, 142, 1-22.

18. Comisarow, M. Fourier Transform Ion Cyclotron Resonance Spectroscopy. Adv. Mass Spectrom. 1978, 7B, 1042-1046.

19. Mitchell, D. W.; Smith, R. D. Prediction of a Space Charge Induced Upper Molecular Mass Limit towards Achieving Unit Mass Resolution in Fourier Transform Ion Cyclotron Resonance Mass Spectrometry. J. Mass Spectrom. 1996, 31, 771-790.

20. Bruce, J. E.; Anderson, G. A.; Lin, C.-Y.; Gorshkov, M.; Rockwood, A. L.; Smith, R. D. A Novel High-Performance Fourier Transform Ion Cyclotron Resonance Cell for Improved Biopolymer Characterization. J. Mass Spectrom. 2000, 35, 85-94.

21. Vartanian, V. H.; Hadjarab, F.; Laude, D. A. Open Cell Analog of the Screened Trapped-Ion Cell Using Compensation Electrodes for Fourier Transform Ion Cyclotron Resonance Mass Spectrometry. Int. J. Mass Spectrom. Ion Process. 1995, 151, 175-187.

22. Barlow, S. E.; Tinkle, M. D. "Linearizing" an Ion Cyclotron Resonance Cell. Rev. Sci. Instrum. 2002, 73, 4185-4200.

23. Wang, M.; Marshall, A. G. A "Screened" Electrostatic Ion Trap for Enhanced Mass Resolution, Mass Accuracy, Reproducibility, and Upper Mass Limit in Fourier-Transform Ion Cyclotron Resonance Mass Spectrometry. Anal. Chem. 1989, 61, 1288-1293.

24. Jackson, G. S.; White, F. M.; Guan, S.; Marshall, A. G. Matrix-Shimmed Ion Cyclotron Resonance Ion Trap Simultaneously Optimized for Excitation, Detection, Quadrupolar Axialization, and Trapping. J. Am. Soc. Mass Spectrom. 1999, 10, 759-769.

25. Rempel, D. L.; Ledford, E. B., Jr.; Huang, S. K.; Gross, M. L. Parametric Mode Operation of a Hyperbolic Penning Trap for Fourier Transform Mass Spectrometry. Anal. Chem. 1987, 59, 2527-2532.

26. Guan, S.; Wahl, M. C.; Wood, T. D.; Marshall, A. G. Enhanced Mass Resolving Power, Sensitivity, and Selectivity in Laser Desorption Fourier Transform Ion Cyclotron Resonance Mass Spectrometry by Ion Axialization and Cooling. Anal. Chem. 1993, 65, 1753-1757.

27. Shi, S. D. H.; Hendrickson, C. L.; Marshall, A. G. Counting Individual Sulfur Atoms in a Protein by Ultrahigh-Resolution Fourier Transform Ion Cyclotron Resonance Mass Spectrometry: Experimental Resolution of Isotopic Fine Structure in Proteins. Proc. Natl. Acad. Sci. U.S.A. 1998, 95, 11532-11537.

28. Winger, B. E.; Hofstadler, S. A.; Bruce, J. E.; Udseth, H. R.; Smith, R. D. High-Resolution Accurate Mass Measurements of Biomolecules Using a New Electrospray Ionization Ion Cyclotron Resonance Mass Spectrometer. J. Am. Soc. Mass Spectrom. 1993, 4, 566-577.

29. Sack, T. M.; Gross, M. L. Pulsed Valve Interface for Gas Chromatography/Fourier Transform Mass Spectrometry. Anal. Chem. 1983, 55, 2419-2421. 
30. Hogan, J. D.; Laude, D. A., Jr. Suspended Trapping Procedure for Alleviation of Space Charge Effects in Gas Chromatography/FourierTransform Mass Spectrometry. Anal. Chem. 1990, 62, 530-535.

31. Dunbar, R. C.; Weddle, G. H. Ion Cyclotron Resonance Time-of-Flight Spectroscopy. Kinetic Energy of p-Iodotoluene Photodissociation Fragment Ions. J. Phys. Chem. 1988, 92, 5706-5709.

32. Wilcox, B. E. Hendrickson, C. L.; Marshall, A. G. Improved Ion Extraction from a Linear Octopole Ion Trap: SIMION Analysis and Experimental Demonstration. J. Am. Soc. Mass Spectrom. 2002, 13, 1304-1312.

33. Mitchell, D. W. Realistic Simulation of the Ion Cyclotron Resonance Mass Spectrometer Using a Distributed Three-Dimensional Particle-inCell Code. J. Am. Soc. Mass Spectrom. 1999, 10, 136-152.

34. Tinkle, M. D.; Barlow, S. E. Image Charge Forces Inside Conducting Boundaries. J. Appl. Phys. 2001, 90, 1612-1624.

35. Marshall, A. G. Milestones in Fourier Transform Ion Cyclotron Resonance Mass Spectrometry Technique Development. Int. I. Mass Spectrom. 2000, 200, 331-356.

36. Bloom, M.; Riggin, M. Theory of Ion Cyclotron Resonance. Can. J. Phys. 1974, 52, 436-455.

37. Vartanian, V. H.; Laude, D. A. Motional Averaging of Ions for Control of Magnetron Motion in Fourier Transform Ion Cyclotron Resonance OpenGeometry Trapped-Ion Cells. Int. J. Mass Spectrom. 1998, 178, 173-186.

38. Kaiser, N. K.; Bruce, J. E. Observation of Increased Ion Cyclotron Resonance Signal Duration through Electric Field Perturbations. Anal. Chem. 2005, 77, 5973-5981.

39. Kim, S.; Choi, M. C.; Kim, S. Hur, M. Kim, H. S.; Yoo, J. S.; Blakney, G. T.; Hendrickson, C. L.; Marshall, A. G. Modification of Trapping Potential by Inverted Sidekick Electrode Voltage during Detection to Extend Time-Domain Signal Duration for Significantly Enhanced Fourier Transform Ion Cyclotron Resonance Mass Resolution. Anal. Chem. 2007, 79, 3575-3580

40. Kaiser, N. K.; Bruce, J. E. Reduction of Ion Magnetron Motion and Space Charge Using Radial Electric Field Modulation. Int. J. Mass Spectrom. 2007, 265, 271-280.
41. Gillig, K. J.; Bluhm, B. K.; Russell, D. H. Ion Motion in a Fourier Transform Ion Cyclotron Resonance Wire Ion Guide Cell. Int. J. Mass Spectrom. Ion Process. 1996, 157/158, 129-147.

42. Solouki, T.; Gillig, K. J.; Russell, D. H. Detection of High-Mass Biomolecules in Fourier Transform Ion Cyclotron Resonance Mass Spectrometry: Theoretical and Experimental Investigations. Anal. Chem. 1994, 66, 1583-1587.

43. Caravatti, P.; Allemann, M. The Infinity Cell: A New Trapped-Ion Cell with Radiofrequency Covered Trapping Electrodes for Fourier Transform Ion Cyclotron Resonance Mass Spectrometry. Org. Mass Spectrom. 1991, 26, 514-518.

44. Anderson, G. A.; Bruce, J. E.; Smith, R. D. ICR-2LS, Richland, WA 1996.

45. Bresson, J. A.; Anderson, G. A.; Bruce, J. E.; Smith, R. D. Improved Isotopic Abundance Measurements for High Resolution Fourier Transform Ion Cyclotron Resonance Mass Spectra via Time-Domain Data Extraction. J. Am. Soc. Mass Spectrom. 1998, 9, 799-804.

46. Du, Y.; Parks, B. A.; Sohn, S.; Kwast, K. E.; Kelleher, N. L. Top-Down Approaches for Measuring Expression Ratios of Intact Yeast Proteins Using Fourier Transform Mass Spectrometry. Anal. Chem. 2006, 78, 686-694.

47. Francl, T. J.; Sherman, M. G.; Hunter, R. L.; Locke, M. J.; Bowers, W. D.; Mclver, R. T., Jr. Experimental Determination of the Effects of Space Charge on Ion Cyclotron Resonance Frequencies. Int. J. Mass Spectrom. Ion Process. 1983, 54, 189-199.

48. Ledford, E. B., Jr.; Rempel, D. L.; Gross, M. L. Space Charge Effects in Fourier Transform Mass Spectrometry Mass Calibration. Anal. Chem. 1984, 56, 2744-2748.

49. Dunbar, R. C.; Chen, J. H.; Hays, J. D. Magnetron Motion of Ions in the Cubical ICR Cell. Int. J. Mass Spectrom. Ion Process. 1984, 57, 39-56.

50. Easterling, M. L.; Mize, T. H.; Amster, I. J. Routine Part-per-Million Mass Accuracy for High-Mass Ions: Space-Charge Effects in MALDI FT-ICR. Anal. Chem. 1999, 71, 624-632. 